\title{
Is the hot appendix really hot?
}

\author{
M.S. Davies, Sally Cunningham, D.A.P. Cooke, D.R. Donaldson and \\ M.H. Thomas
}

Department of Surgery, St Peter's Hospital, Chertsey, Surrey, UK

\begin{abstract}
Summary: The skin temperature in the right and left iliac fossae was recorded in 100 patients who were referred with a provisional diagnosis of acute appendicitis. This was not found to be a reliable aid in diagnosis of acute appendicitis nor an indicator to the need for surgery in patients with right iliac fossa pain.
\end{abstract}

\section{Introduction}

It has been suggested that measurement of the temperature in the right iliac fossa may help in the diagnosis of acute appendicitis. ${ }^{1}$ In a cohort of patients undergoing appendicectomy, a temperature difference of greater than one degree Celsius between the right and left iliac fossae was found to have a specificity and positive predictive value of $100 \%$ in patients with acute appendicitis. ${ }^{2}$ A study using microwave thermography on 72 patients showed a specificity of $70 \%$ and sensitivity of $84 \%$ in the diagnosis of acute appendicitis. ${ }^{3}$ However, Steele, in 1986, concluded that thermography was not of diagnostic value in this condition. ${ }^{4}$

This prospective single-blind study was conducted to investigate whether measurement of the difference between the right and left iliac fossa skin temperatures could help in making the decision to operate.

\section{Materials and methods}

One hundred consecutive patients referred by the casualty officer or by a general practitioner with a provisional diagnosis of acute appendicitis were studied. The initial examination was performed by the house surgeon who measured the skin temperature at McBurney's point and an equivalent point in the left iliac fossa with a liquid crystal strip thermometer (Feverscan; Robinson, Chesterfield, UK). This device measures temperature in one degree Celsius increments and is accurate to \pm 0.3 degrees Celsius (manufacturer's information

Correspondence: M.S. Davies, F.R.C.S., Department of Orthopaedics, Charing Cross Hospital, Fulham Palace Road, London SW6 8RF, UK.

Accepted: 5 July 1993 sheet). In an attempt to standardize the condition of measurement, the temperature readings were taken immediately after exposing the abdomen. The test was considered positive if the right iliac fossa temperature was one degree Celsius or more higher than on the left and negative if equal to or less than the temperature on the left. The decision to operate was made by the registrar who was unaware of the skin temperature readings.

\section{Results}

The results of the study are summarized in Figure 1 Sixty patients underwent surgery and the histology was recorded. The remaining 40 patients were discharged within 48 hours without operative intervention and the assumption was made that these patients did not have appendicitis. Where possible, an alternative diagnosis was made.

In nine (of 17) patients, in whom a positive temperature was found, acute appendicitis was confirmed by histology. Four other patients with a positive test were operated upon and the findings were an ovarian cyst, mesenteric adenitis, pelvic inflammatory disease, with no cause being found in one. The remaining four with a positive test did not have an operation and their symptoms resolved.

A negative temperature test was recorded in 47 (of 60 ) patients undergoing surgery and $70 \%$ of these had acute appendicitis confirmed. The remaining patients had ovarian cysts (two), pelvic inflammatory disease (three), terminal ileitis (one), faecolith (one) and no cause was found in three.

Nine patients had a skin temperature higher in the left iliac fossa than the right. Three had acute appendicitis, two had enterobius infestation of the appendix, one was constipated and in the remainder no cause for the right iliac fossa pain was found. 


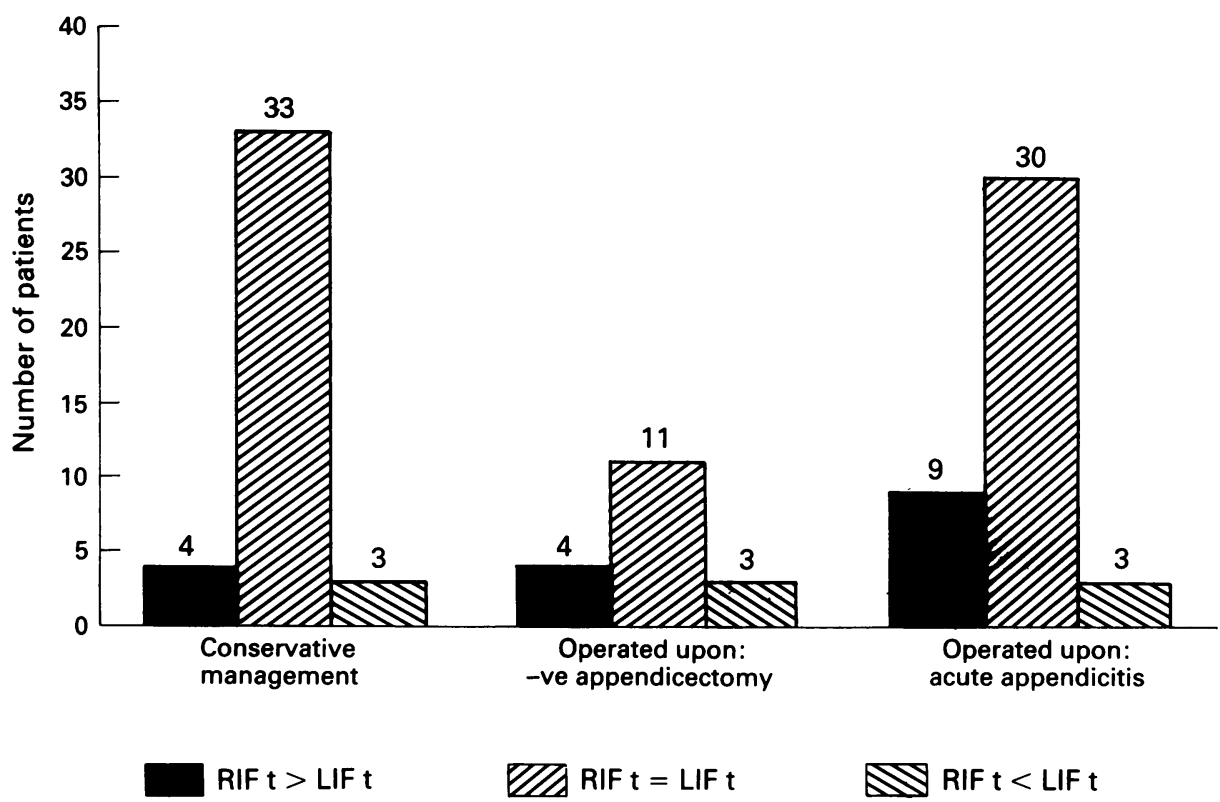

Figure 1 Management and findings in 100 patients referred with a provisional diagnosis of acute appendicitis, grouped according to the skin temperature test. $\mathrm{RIF} t=$ right iliac fossa temperature; $\mathrm{LIF} t=$ left iliac fossa temperature; - ve = negative.

In only one patient (of 100) was there a temperature difference greater than one degree Celsius between the right and left iliac fossae. The patient was a 10 year old girl who underwent a negative appendicectomy.

In considering the value of the test in diagnosing acute appendicitis, the results show a sensitivity of $21.4 \%$ and a specificity of $86.2 \%$, with a positive predictive value of $52.9 \%$.

\section{Discussion}

It is well known that acute appendicitis can be a difficult diagnosis to make. A simple and reliable noninvasive test for acute appendicitis would be useful both to the referring doctor and to the surgeon who has to make the decision whether or not to operate. The aim of this study was to determine whether measurement of the difference between the skin temperatures in the right and left iliac fossae, using a liquid crystal thermometer, would provide such a test.

The results showed a sensitivity of $21.4 \%$, a specificity of $86.2 \%$ and a positive predictive value of $52.9 \%$ in making a diagnosis of acute appen- dicitis. One could accept a false positive result if it indicated the need for surgical intervention for pathology other than appendicitis, but this was not the case.

Despite attempting to standardize the conditions of measurement by performing the test immediately after exposing the abdomen, clearly it would not have been feasible to maintain a constant room temperature in the accident and emergency department (where the measurements were made) for the duration of the study. Indeed, for the test to be useful to referring doctors as well as surgeons it would have to be independent of room temperature. Analysis of the results showed there to be no significance in the actual skin temperature of the right iliac fossa, that is, local hyperthermia was not found to correlate with pathology.

We conclude that neither the measurement of right iliac fossa skin temperature, nor the measurement of the difference in skin temperature between the right and left iliac fossae is a reliable aid to the diagnosis of acute appendicitis. Furthermore, neither of these measurements provides an indication that a patient with right iliac fossa pain needs surgery. 


\section{References}

1. Zakharov, S.N., Svitich, I.M. \& Baskakov, V.A. Electrothermometry in the diagnosis of destructive forms of acute appendicitis and acute cholecystitis. Khirugia (Sofia) 1981, 10: 37-39.

2. Hambridge, J.E. Use of skin thermometer to diagnose acute appendicitis. Br Med J 1990, 300: 722.
3. Stallard, S., Land, D.V. \& George, W.D. Microwave thermography in the diagnosis of acute appendicitis. Br J Surg 1987, 74: 1150.

4. Steele, R.J. Abdominal thermography in acute appendicitis. Scott Med J 1986, 31: 299-300. 